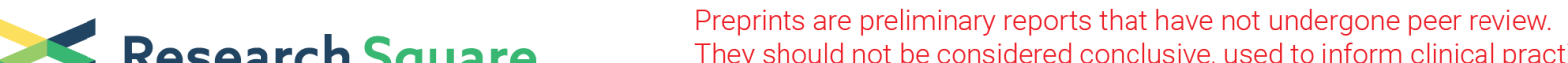 $\begin{array}{ll}\text { Research Square } & \text { They should not be considered conclusive, used to inform clinical practice, } \\ \text { or referenced by the media as validated information. }\end{array}$
}

\section{A New Minimally Invasive Surgery of Hallux Valgus: Technique and Preliminary Outcomes}

\section{Changjun Guo}

Shanghai Jiao Tong University Medical School Affiliated Ruijin Hospital https://orcid.org/0000-00034151-5150

\section{Xingchen Li}

Shanghai Jiao Tong University Medical School Affiliated Ruijin Hospital

\section{Chunguang Li}

Shanghai Jiao Tong University Medical School Affiliated Ruijin Hospital

\section{Yang Xu}

Shanghai Jiao Tong University Medical School Affiliated Ruijin Hospital

\section{Ming Cai}

Shanghai Jiao Tong University Medical School Affiliated Ruijin Hospital

Xiangyang Xu ( $\sim$ xu664531@126.com)

Shanghai Jiao Tong University School of Medicine https://orcid.org/0000-0001-9194-4901

\section{Research}

Keywords: hallux valgus, chevron, akin, minimally invasive surgery

Posted Date: August 12th, 2020

DOI: https://doi.org/10.21203/rs.3.rs-54804/v1

License: (c) (i) This work is licensed under a Creative Commons Attribution 4.0 International License.

Read Full License 


\section{Abstract}

\section{Purpose}

This study aimed to present a new minimally invasive surgery (MIS) technique and evaluate the safety and efficacy for correction of hallux valgus deformity at a preliminary follow-up.

\section{Methods}

48 consecutive feet that underwent a new MIS of hallux valgus with a mean 17.6 months follow-up (range 9 to 28 months). This technique uses a new osteotomy of the first metatarsal with screw fixation and percutaneous Weil osteotomy of lesser metatarsal without fixation. All patients were clinically evaluated using the Manchester-Oxford Foot Questionnaire (MOXFQ), American Orthopedic Foot \& Ankle Society Hallux Metatarsophalangeal-Interphalangeal score (AOFAS HMI) and visual analogue scale (VAS). Radiographic measures included hallux valgus angle (HVA), intermetatarsal angle (IMA) and the length of the first metatarsal.

\section{Results}

There was significant improvement in the radiographic parameters $(P<0.001)$. The IMA decreased from $13.3^{\circ}$ to $9.8^{\circ}$, the HVA improved from $35.9^{\circ}$ to $17.9^{\circ}$ and the mean MT length decreased by $4 \mathrm{~mm}$. There was significant improvement of VAS in the postoperative 2 weeks $(P<.001)$. There were significant improvements in the MOXFQ scores and AOFAS HMI scores, respectively. The total rate of complication was $10.4 \%$.

\section{Conclusion}

The preliminary radiographic and clinical outcomes of this new minimally invasive surgery show that it is a safe procedure and had significantly less pain in the first 2 weeks following surgery.

\section{Introduction}

Percutaneous and minimally invasive surgery (MIS) for hallux valgus is increasingly common in orthopedics by reason of the inherent for smaller scars, lesser postoperative pain, rapid recovery, reduced rehabilitation times, and decreased risk of infection [1,2].

Initially, percutaneous hallux valgus technique was accomplished applying Isham[3], Bosch or ReverdinIsham procedures[4] and modifications of these, which consist of arthroscopic assistant technique, the Endolog technique, percutaneous extra-articular reverse-L Chevron (PERC) and percutaneous Chevron/Akin osteotomies[5-8] . The method of fixation is a critical aspect for the surgical correction of hallux valgus. First-generation percutaneous technique was reported by Isham[3] and no internal fixation after the osteotomy. Later, the second generation of improvement was carried out, which included a distal 
transverse osteotomy of the first metatarsal fixed with an axial wire [8,9]. At present, in order to enhance the stability, screw fixation was carried out [10].

To date, many studies have evaluated the results of MIS techniques and open therapeutic surgeries for hallux valgus $[8,11]$. Meanwhile, systematic reviews have been reported to estimate the results of MIS techniques for treating the hallux valgus $[1,12]$. Those reviews pinpointed limitations of the achievable evidence to determine clear proposals for MIS in hallux valgus correction. There were too few studies on individual surgical technique to determine whether one is more powerful than another one.

Because of the lack of evidence that which technique is more superior, we have implemented a new MIS osteotomy and adopted the screw fixation method. In order to accept a new operative technique, it is essential to see clinical outcomes. The aim of this study was to present the radiographic correction, complications, postoperative pain levels and clinical outcomes at a preliminary follow-up.

\section{Methods}

This retrospective cohort study was approved by institutional review board. Patients were enrolled if they had painful hallux valgus and failed conservative treatment. The exclusion criteria were patients had peripheral vascular disease, neuromuscular disease, rheumatoid arthritis and recurrent hallux valgus.

We firstly enrolled 56 consecutive feet underwent the MIS technique of hallux valgus. Patients with recurrent hallux valgus ( 2 feet) and lost follow-up ( 6 feet) were excluded. Finally, 48 feet (41 patients) that underwent a percutaneous MIS of hallux valgus were enrolled. The baseline characteristics are shown in Table 1.

Table 1

Patient Demographics.

\begin{tabular}{|ll|}
\hline Demographics & \\
\hline Sex, male/female & $3 / 45$ \\
\hline Age, mean $\pm \mathrm{SD}, \mathrm{y}$ & $60.9 \pm 12.2$ \\
\hline Body mass index, & $22.5 \pm 2.96$ \\
\hline mean $\pm \mathrm{SD}, \mathrm{kg} / \mathrm{m} 2$ & \\
\hline
\end{tabular}

Weightbearing anteroposterior (AP) and lateral radiographs of the foot were collected for all patients to evaluate the severity of the deformity. The hallux valgus angle (HVA), intermetatarsal angle (IMA) and first metatarsal (MT) length were assessed as the radiological parameters preoperatively and postoperatively at last follow-up. Radiographic measurement was performed by a single observer (CJ, G). The visual analogue scale (VAS) was collected preoperatively and on 2 weeks follow-up postoperatively. Clinical outcomes were measured by American Orthopedic Foot \& Ankle Society Hallux Metatarsophalangeal-Interphalangeal (AOFAS HMI) score, and Manchester-Oxford Foot Questionnaire 
(MOXFQ) [13] preoperatively and postoperatively at the last follow-up. Clinical outcome was evaluated in terms of passive range of motion (ROM) of the first metatarsophalangeal joint (MTPJ) and the stiffness of the first MTPJ was defined as the ROM $<30^{\circ}$. All the clinical complications including wound problem, numbness, nonunion, and revision were collected at the last follow-up.

\section{Technique}

\section{Minimally invasive surgery (MIS)}

The patient was performed using regional anesthesia or general anesthesia without a tourniquet in a supine position.

A medial incision was used just proximal to the first metatarsal head, and the medial eminence was excised with a $2 \star 20 \mathrm{~mm}$ burr and the bone debris was discharged. We always rinsed the incision and burr with ice sterile saline to prevent the burn complication during the osteotomy. The speed of the burr was not exceeding $3000 \mathrm{rpm}$ to avoid thermal necrosis of the skin and bone.

The starting point of osteotomy was at about $5 \mathrm{~mm}$ proximal to the first metatarsal head, and the direction of osteotomy was from the distal to the proximal. From the lateral view, it was plantarization about 45 degrees (Figure 1a, Figure2). After the osteotomy finished, we used a scissor to release the lateral soft tissue through the osteotomy line.

When the distal osteotomy was completely loosened, we manually reduced the osteotomy. One or two the hollow nail guidewires were inserted through separate incisions in order to fix the osteotomy, and the position was verified fluoroscopically and adjusted accordingly (Figure 3a,3b). After the position was satisfactory, one or two 2.5mm-diameter hollow headless nails (Wright, USA) were selected for fixation (Figure 3c,3d).

An Akin osteotomy was performed if there was residual hallux valgus after the first metatarsal osteotomy. This was undertaken through a dorsomedial approach, using a 1.5 * $20 \mathrm{~mm}$ burr (Figure 1b), and one screw fixation after the akin osteotomy (Figure 3c,3d).

The osteotomy of the 2-5 metatarsals depends on the preoperative symptoms and the length of the 2-5 metatarsals after the first metatarsal osteotomy and fixation. The burr of these osteotomies is 1.5 * $20 \mathrm{~mm}$, without screw fixation (Figure 1c).

Postoperatively, the hallux was mobilized in 8-shaped bandage and the patients were allowed fully weightbearing immediately in a hard-bottom shoe (Darco, USA) without the aid of crutches. Patients were encouraged to elevate the foot in the first 2 weeks after surgery to reduce the swelling. The shoe was removed after 6 weeks and patients were encouraged to wear a pair of sneakers. 


\section{Statistical Analysis}

Power analysis was carried out to guarantee that the study sample size was adequate. All the continuous variables are described as means \pm standard deviations. Mann-Whitney $U$ test or Student $t$ test was used for continuous variables, depending on the data distribution. The Matched-pairs $t$ test was used to analyze any significant difference between the preoperative and postoperative clinical outcomes. The alpha level was set at less than .05. Statistical analyses were using Stata statistical software (version 15, StataCorp, College Station, TX).

\section{Results}

\section{Radiologic Outcomes}

Compared with preoperative radiographic outcomes, there were significant differences in the HVA and IMA at the final follow-up $(P<.05)$. The IMA decreased from $13.3^{\circ}$ to $9.8^{\circ}$ and the HVA improved from $35.9^{\circ}$ to $17.9^{\circ}$. The Mean MT length decreased by $4 \mathrm{~mm}(P<.05)$. Table 2 summarizes the radiographic outcomes.

\section{Clinical Outcomes}

\section{Clinical}

The mean follow-up was 17.6 months (range 9 to 28 months). 25 feet required Akin osteotomy and 30 feet required Weil osteotomies of lesser metatarsal. The MIS achieved significant correction of the hallux deformity (Figure 4 and Figure 5). The walking score improved from 60.2 to $13.5(P<.001)$, the pain score from 54.5 to $15.8(P<.001)$, and the social interaction score from 56.9 to $13.3(P<.001)$.

The AOFAS HMI score improved significantly improved from 50.6 to 85.2 postoperatively $(P<.001)$. The VAS score statistically significant improvement in the postoperative 2 weeks $(P<.001)$. Table 2 summarizes the clinical outcomes. 
Table 2

Summary of Clinical outcome and Radiological outcome of the MIS.

\begin{tabular}{|lllll|}
\hline Variables & & Mean & SD & Pvalue \\
\hline Follow-up(months) & & 17.6 & 6.4 & \\
\hline Radiological outcomes & & & & \\
\hline HVA $\left(^{\circ}\right)$ & Preoperative & 35.9 & 10.1 & $<0.001$ \\
\hline & Postoperative & 17.9 & 9.3 & \\
\hline IMA $\left(^{\circ}\right)$ & Preoperative & 13.3 & 3.3 & $<0.001$ \\
\hline MT length(cm) & Postoperative & 9.8 & 3.8 & \\
\hline Clinical outcomes & Preoperative & 5.0 & 0.2 & $<0.001$ \\
\hline MOXFQ scores & Postoperative & 4.6 & 0.2 & \\
\hline Walking & & & & \\
\hline & & & & \\
\hline Pain & Preoperative & 60.2 & 9.1 & $<0.001$ \\
\hline VAS & Postoperative & 13.5 & 7.8 & \\
\hline Social Interaction & Preoperative & 54.5 & 10.5 & $<0.001$ \\
\hline & Postoperative & 15.8 & 13.3 & \\
\hline & Preoperative & 56.9 & 10.7 & $<0.001$ \\
\hline & Postoperative & 13.3 & 10.9 & \\
\hline & Preoperative & 50.6 & 12.4 & $<0.001$ \\
\hline & Postoperative & 85.2 & 13.8 & \\
\hline & Preoperative & 7.63 & 1.1 & $<0.001$ \\
\hline & & & 0.9 & \\
\hline & & & & \\
\hline
\end{tabular}

\section{Complications}

There were 1 burn wound problems, 1 screw irritation, 1 revision, 1 patient with paresthesia and 1 stiffness of the first MTPJ.There was no avascular necrosis, nonunion, hallux varus, metatarsalgia or recurrence. The total rate of complication was $10.4 \%$.

\section{Discussion}


This new MIS technique is a third-generation technique of percutaneous hallux valgus correction in which it uses the stable internal fixation. There are a few reports on the third-generation percutaneous hallux valgus surgery technique. Jowett [14] reported a prospective study of 78 patients with a mean follow-up of 25 months. The AOFAS score and the radiographic parameters (HVA and IMA) had improved significantly. Lee et al [11] reported a prospective randomized study of 25 percutaneous Chevron/akin (PECA) versus 25 open technique cases with 6 months of follow-up. They reported that both groups demonstrated comparable good to excellent clinical and radiologic results at last follow-up. However, the PECA group had significantly lesser pain in the first 6 weeks after surgery. Brogan et al [8] reported a retrospective cohort study of 81 cases (49 MIS and 32 open distal chevron osteotomies) with a minimum 24 months of follow-up, and reported that both groups demonstrated comparable clinical and radiologic results of mild-moderate hallux valgus. Lucas y Hernandez [7] in their series of 53 cases of hallux valgus correction using the percutaneous extra-articular reverse-L Chevron (PERC) technique with a mean followup of 60 months revealed a main improvement of good first MTPJ range of motion underwent this technique. Vernois and Redfern[15] reported a case series of 341 patients with a minimum 12 months of follow-up, and reported HVA and IMA had improved significantly and the excellent or good satisfaction rates were $95 \%$. Holme[16] reported a case series of 40 patients with a 12 months of follow-up, and reported the MOXFQ score and the AOFAS score had improved significantly.

The current study found similar clinical and radiologic outcomes compared to previous studies. However, our technique has some discrepancies from previous reported literatures. First, we use an oblique metatarsal osteotomy, which is different from transverse metatarsal osteotomy and Chevron osteotomy. We can both rotate the distal osteotomy block and correct distal metatarsal articular angle (DMAA) simultaneously after osteotomy. DMAA has not been measured in our cases, because there is a poor interobserver reliability [17]and is not frequently used in research due to this reason [1].

Second, we use one or two $2.5 \mathrm{~mm}$-diameter headless hollow screws to fix the osteotomy in order to provide the stability and allow fully weight-bearing immediately after surgery in a hard-bottom shoe without the aid of crutches. An eminent complication of the Bosch technique is decreased range of motion or stiffness of the first MTPJ by reason of the fact which a K-wire is fixed in the toe for four weeks at least, and there is no exercise of the joint [1]. However, many studies have no report of the postoperative range of motion of the first MTPJ.The first MTPJ can be exercised due to immediate weight-bearing in our technique and only one case had the MTPJ stiffness.

Third, there are some considerations about the experience the osteotomy is created with a $2 \mathrm{~mm}$-diameter burr that can generate relative shortening of the first ray, and lead to transfer metatarsalgia[18].Our results show the mean MT length decreased by $4 \mathrm{~mm}$, and the potential increased need for lesser metatarsal shorting due to first metatarsal shorting. We perform the osteotomy of the lesser metatarsals depending on the preoperative symptoms and the length of the lesser metatarsals after the first metatarsal osteotomy. Our theory is that the position of 2-5 metatarsals after the osteotomy using $1.5 \mathrm{~mm}$-diameter burr without fixation can be adjusted automatically after the weight-bearing. The need 
for additional surgery can add to risk to the patient, however, there were no cases of transfer metatarsalgia or nonunion of the osteotomy of lesser metatarsals.

Safeness and complications were important concern, because the procedures were performed without explicit view for the structures. For the reason of high rate of major complications, a previous prospective study evaluating percutaneous hallux valgus surgery was abandoned after 3 months during early followup[19].The wound problems coming from burning of the skin with the high speed of burr after minimal invasive hallux valgus surgery varied from $0-13 \%$ in beginners during the learning curve[20]. In our early cases, we found one case with skin burning, and then we rinsed the incision and burr with ice sterile saline and low-speed burr to reduce the risk of thermal damage. Consequently, no burn complication was recorded after these measures. The rates of the complications were diverse widely even within the same technique. The Bosch technique was reported to have $0 \%$ complication rates [21] or $22 \%$ [22] or $27.2 \%$ [23]. The Reverdin- Isham technique was reported to vary from a 5\% [24] to a 73\% [25] rate of complications. The Endolog technique was reported to have a range from 0 to $10 \%$ complication rates and in the distal soft tissue release group from 0 to $4 \%$ of cases [12]. During the third-generation technical literatures, the rate of complication was also varied from a $24 \%$ [11] or $15.5 \%$ [7] or $14 \%$ [14] or $10 \%$ [16] or $2 \%[15]$. It is possible that a low complication rate might not have included the complications such as stiffness or prominent screws in papers. Our total rate of complication was $10.4 \%$.

There are several limitations to this study. First, a relatively small number of participants in a limited time period are enrolled and the follow-up period is relatively short. It is important to recognize the long-term clinical outcomes of a new operative technique, and it is apparently true that the absolute benefits of MIS are in the early postoperative period and long-term data collection, including recurrence and patient satisfaction, will be evaluated in the future studies. Second, this is a not a randomized control study, it would be excellent to compare the new technique method with several other well-known techniques for more reliable data.

\section{Conclusion}

We conclude that this new third-generation MIS technique is a safe procedure for correcting symptomatic hallux valgus, however longer-term studies with comparison groups should be implemented to further verify MIS for the treatment of hallux valgus.

\section{Abbreviations}

AOFAS HMI: American Orthopedic Foot \& Ankle Society Hallux Metatarsophalangeal-Interphalangeal score;AP :anteroposterior囚DMAA: distal metatarsal articular angle ;HVA :hallux valgus angle;IMA: intermetatarsal angle; MIS:minimally invasive surgery;MOXFQ:Manchester-Oxford Foot Questionnaire; MT: metatarsal; MTPJ: metatarsophalangeal joint; PECA: percutaneous Chevron/akin ;PERC: percutaneous extra-articular reverse-L Chevron;ROM:range of motion; VAS $₫$ visual analogue scale. 


\section{Declarations}

\section{Ethics approval and consent to participate}

Our study was approved by the Ruijin North hospital ethics committee, Shanghai JiaoTong University School of Medicine. Written consent was provided by participants to be included in the study.

\section{Consent for publication}

Written informed consent for publication was obtained from all participants.

\section{Availability of data and materials}

All relevant data are freely available to any scientist wishing to use them for non-commercial purposes, without breaching patient confidentiality.

\section{Competing interests}

The authors have no conflict of interest.

\section{Funding}

This study was supported in part by a research grants from National Natural Science Foundation of China grants (No. 81772372).

\section{Authors' contributions}

Changjun Guo and Xingchen Li carried out the interpretation of the results and drafted the manuscript. Chunguang Li and Yang Xu made substantive intellectual contributions to the analyses and the interpretation of the results. Ming Cai supervised data collection and critically reviewed the manuscript. Xiangyang Xu contributed to drafting and revision of the manuscript. All authors read and approved the final manuscript.

\section{Acknowledgements}

The authors gratefully thank those surgeons and patients who made this study possible.

\section{Authors' information}


1.Department of Orthopaedics, Rui Jin Hospital, Shanghai Jiao Tong University School of Medicine, Shanghai, China. 2.Department of Orthopaedics, Rui Jin North Hospital, Shanghai Jiao Tong University School of Medicine, Shanghai, China.

\section{References}

1. Bia A, Guerra-Pinto F, Pereira BS, Corte-Real N, Oliva XM. Percutaneous Osteotomies in Hallux Valgus: A Systematic Review. J Foot Ankle Surg. 2018;57(1):123-30. doi:10.1053/j.jfas.2017.06.027.

2. Kaufmann G, Dammerer D, Heyenbrock F, Braito M, Moertlbauer L, Liebensteiner M. Minimally invasive versus open chevron osteotomy for hallux valgus correction: a randomized controlled trial. Int Orthop. 2019;43(2):343-50. doi:10.1007/s00264-018-4006-8.

3. Isham SA. The Reverdin-Isham procedure for the correction of hallux abducto valgus. A distal metatarsal osteotomy procedure. Clin Podiatr Med Surg. 1991;8(1):81-94.

4. Bauer T, Biau D, Lortat-Jacob A, Hardy P. Percutaneous hallux valgus correction using the ReverdinIsham osteotomy. Orthop Traumatol Surg Res. 2010;96(4):407-16. doi:10.1016/j.otsr.2010.01.007.

5. Lui TH, Chan KB, Chow HT, Ma CM, Chan PK, Ngai WK. Arthroscopy-assisted correction of hallux valgus deformity. Arthroscopy. 2008;24(8):875-80. doi:10.1016/j.arthro.2008.03.001.

6. Di Giorgio L, Touloupakis G, Simone S, Imparato L, Sodano L, Villani C. The Endolog system for moderate-to-severe hallux valgus. J Orthop Surg (Hong Kong). 2013;21(1):47-50. doi:10.1177/230949901302100113.

7. Lucas y Hernandez J, Golano P, Roshan-Zamir S, Darcel V, Chauveaux D, Laffenetre O. Treatment of moderate hallux valgus by percutaneous, extra-articular reverse-L Chevron (PERC) osteotomy. Bone Joint J. 2016;98-B(3):365-73. doi:10.1302/0301-620X.98B3.35666.

8. Brogan K, Lindisfarne E, Akehurst H, Farook U, Shrier W, Palmer S. Minimally Invasive and Open Distal Chevron Osteotomy for Mild to Moderate Hallux Valgus. Foot Ankle Int. 2016;37(11):1197204. doi:10.1177/1071100716656440.

9. Magnan B, Pezze L, Rossi N, Bartolozzi P. Percutaneous distal metatarsal osteotomy for correction of hallux valgus. J Bone Joint Surg Am. 2005;87(6):1191-9. doi:10.2106/JBJS.D.02280.

10. Brogan $K$, Voller T, Gee $C$, Borbely $T$, Palmer $S$. Third-generation minimally invasive correction of hallux valgus: technique and early outcomes. Int Orthop. 2014;38(10):2115-21. doi:10.1007/s00264-014-2500-1.

11. Lee M, Walsh J, Smith MM, Ling J, Wines A, Lam P. Hallux Valgus Correction Comparing Percutaneous Chevron/Akin (PECA) and Open Scarf/Akin Osteotomies. Foot Ankle Int. 2017;38(8):838-46. doi:10.1177/1071100717704941.

12. Malagelada F, Sahirad C, Dalmau-Pastor M, Vega J, Bhumbra R, Manzanares-Cespedes MC, Laffenetre O. Minimally invasive surgery for hallux valgus: a systematic review of current surgical techniques. Int Orthop. 2019;43(3):625-37. doi:10.1007/s00264-018-4138-x. 
13. Dawson J, Boller I, Doll H, Lavis G, Sharp R, Cooke P, Jenkinson C. Responsiveness of the Manchester-Oxford Foot Questionnaire (MOXFQ) compared with AOFAS, SF-36 and EQ-5D assessments following foot or ankle surgery. J Bone Joint Surg Br. 2012;94(2):215-21. doi:10.1302/0301-620X.94B2.27634.

14. Jowett CRJ, Bedi HS. Preliminary Results and Learning Curve of the Minimally Invasive Chevron Akin Operation for Hallux Valgus. J Foot Ankle Surg. 2017;56(3):445-52. doi:10.1053/j.jfas.2017.01.002.

15. Vernois J, Redfern D. Percutaneous Chevron; the union of classic stable fixed approach and percutaneous technique. Fuß Sprunggelenk. 2013;11(2):70-5. doi:10.1016/j.fuspru.2013.03.001.

16. Holme TJ, Sivaloganathan SS, Patel B, Kunasingam K. Third-Generation Minimally Invasive Chevron Akin Osteotomy for Hallux Valgus. Foot Ankle Int. 2020;41(1):50-6. doi:10.1177/1071100719874360.

17. Chi TD, Davitt J, Younger A, Holt S, Sangeorzan BJ. Intra- and inter-observer reliability of the distal metatarsal articular angle in adult hallux valgus. Foot Ankle Int. 2002;23(8):722-6. doi:10.1177/107110070202300808.

18. Jung HG, Zaret DI, Parks BG, Schon LC. Effect of first metatarsal shortening and dorsiflexion osteotomies on forefoot plantar pressure in a cadaver model. Foot Ankle Int. 2005;26(9):748-53. doi:10.1177/107110070502600913.

19. Kadakia AR, Smerek JP, Myerson MS. Radiographic results after percutaneous distal metatarsal osteotomy for correction of hallux valgus deformity. Foot Ankle Int. 2007;28(3):355-60. doi:10.3113/FAl.2007.0355.

20. de Prado M, Vaquero PLR,J, Golanó P. Tratamiento quirúrgico percutáneo del hallux valgus mediante osteotomías múltiples[Percutaneous hallux valgus repair by multiple osteotomies]. Revista Española de Cirugía Ortopédica y Traumatología. 2003;47(6):406-16. doi:https://doi.org/10.1016/S18884415(03)76145-1.

21. Giannini S, Cavallo M, Faldini C, Luciani D, Vannini F. The SERI distal metatarsal osteotomy and Scarf osteotomy provide similar correction of hallux valgus. Clin Orthop Relat Res. 2013;471(7):2305-11. doi:10.1007/s11999-013-2912-z.

22. Tong C-K, Ho Y-F. Use of Minimally Invasive Distal Metatarsal Osteotomy for Correction of Hallux Valgus. Journal of Orthopaedics Trauma Rehabilitation. 2012;16(1):16-21. doi:10.1016/j.jotr.2011.07.004.

23. Siddiqui NA, LaPorta G, Walsh AL, Abraham JS, Beauregard S, Gdalevitch M. Radiographic Outcomes of a Percutaneous, Reproducible Distal Metatarsal Osteotomy for Mild and Moderate Bunions: A Multicenter Study. J Foot Ankle Surg. 2019;58(6):1215-22. doi:10.1053/j.jfas.2019.04.012.

24. 10.1016/j.ocl.2009.05.002

Bauer T, de Lavigne C, Biau D, De Prado M, Isham S, Laffenetre O. (2009) Percutaneous hallux valgus surgery: a prospective multicenter study of 189 cases. Orthop Clin North Am 40 (4):505-514, ix. doi:10.1016/j.ocl.2009.05.002. 
25. Gicquel T, Fraisse B, Marleix S, Chapuis M, Violas P. Percutaneous hallux valgus surgery in children: short-term outcomes of 33 cases. Orthop Traumatol Surg Res. 2013;99(4):433-9. doi:10.1016/j.otsr.2013.02.003.

\section{Figures}
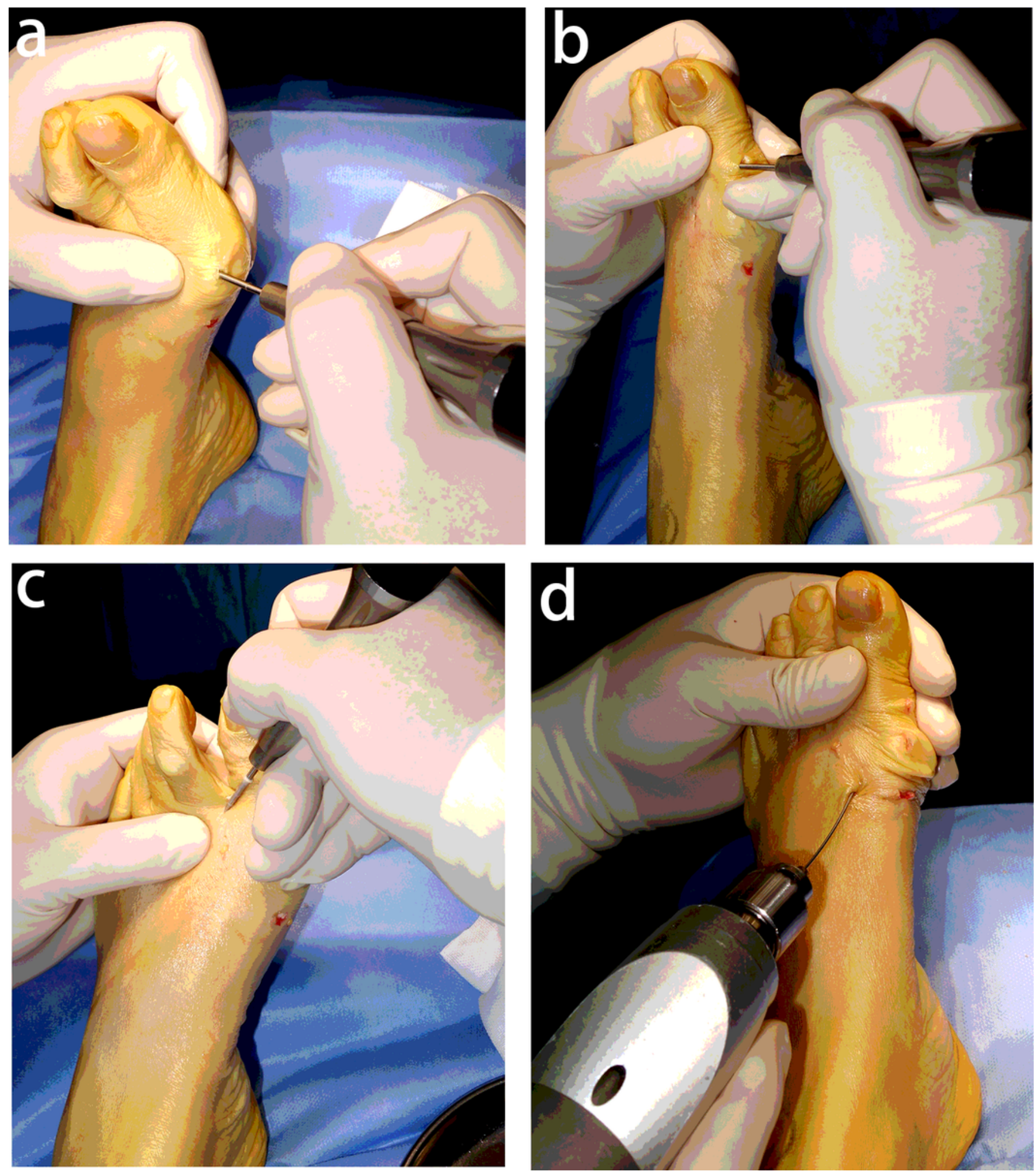

Figure 1 
(a): The starting point of osteotomy and the direction of osteotomy was from the distal to the proximal ;

(b): An Akin osteotomy was undertaken through a dorsomedial approach using a $1.5 * 20 \mathrm{~mm}$ burr;(c): The burr of these osteotomies is $1.5 * 20 \mathrm{~mm}$ without screw fixation;(d): One or two the hollow nail guidewires were inserted through separate incisions in order to fix the osteotomy.

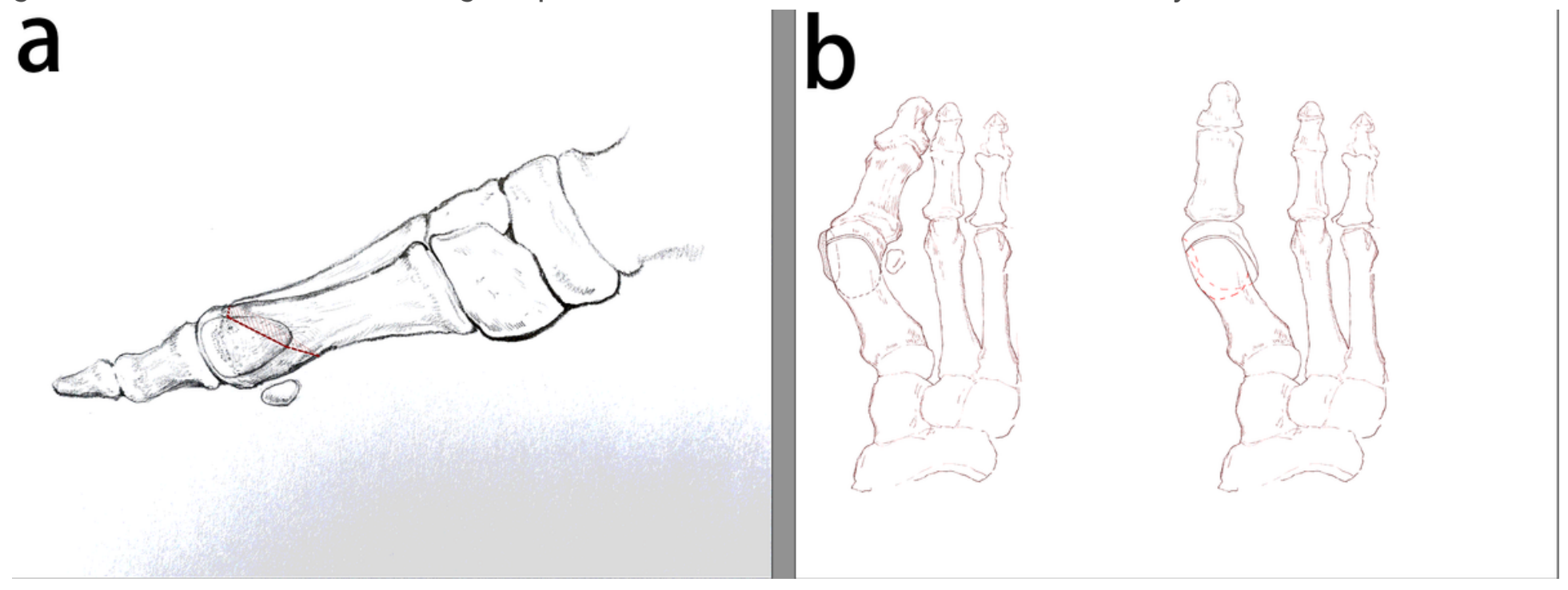

Figure 2

Schematic drawings of the MIS osteotomy. (a): lateral view; From the lateral view, it was plantarization about 45 degrees;(b) A-P view: the distal osteotomy was completely and reduced the osteotomy manually. 


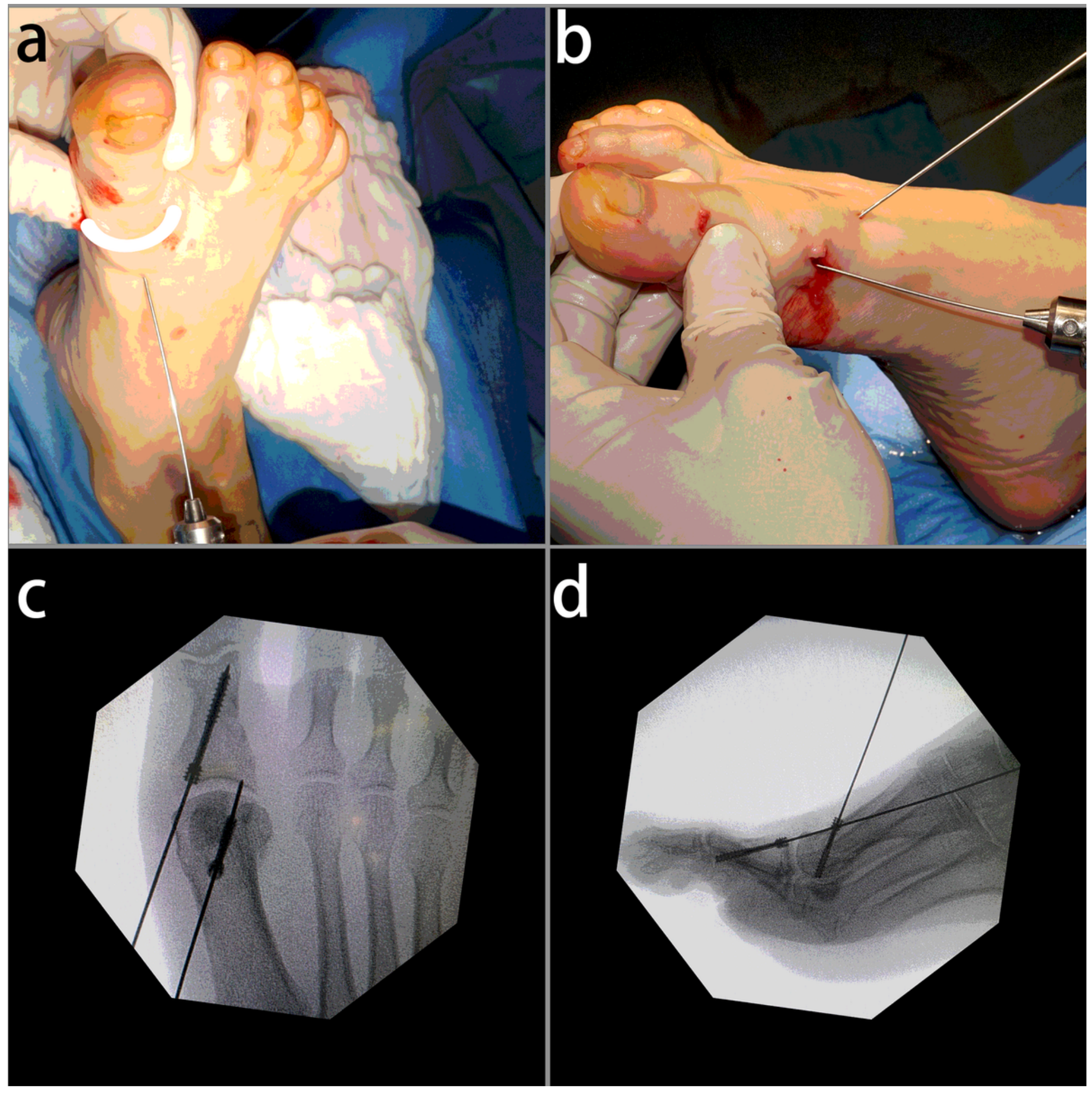

\section{Figure 3}

(a): Clinical photo showing the correction maneuver of the deformity (force applied as demonstrated with arrow). (b): Applying hollow nail guidewires for fixation of the fragment. Radiography showing placement of the screw percutaneously after osteotomy. (c): A-P view;(d): lateral view. 


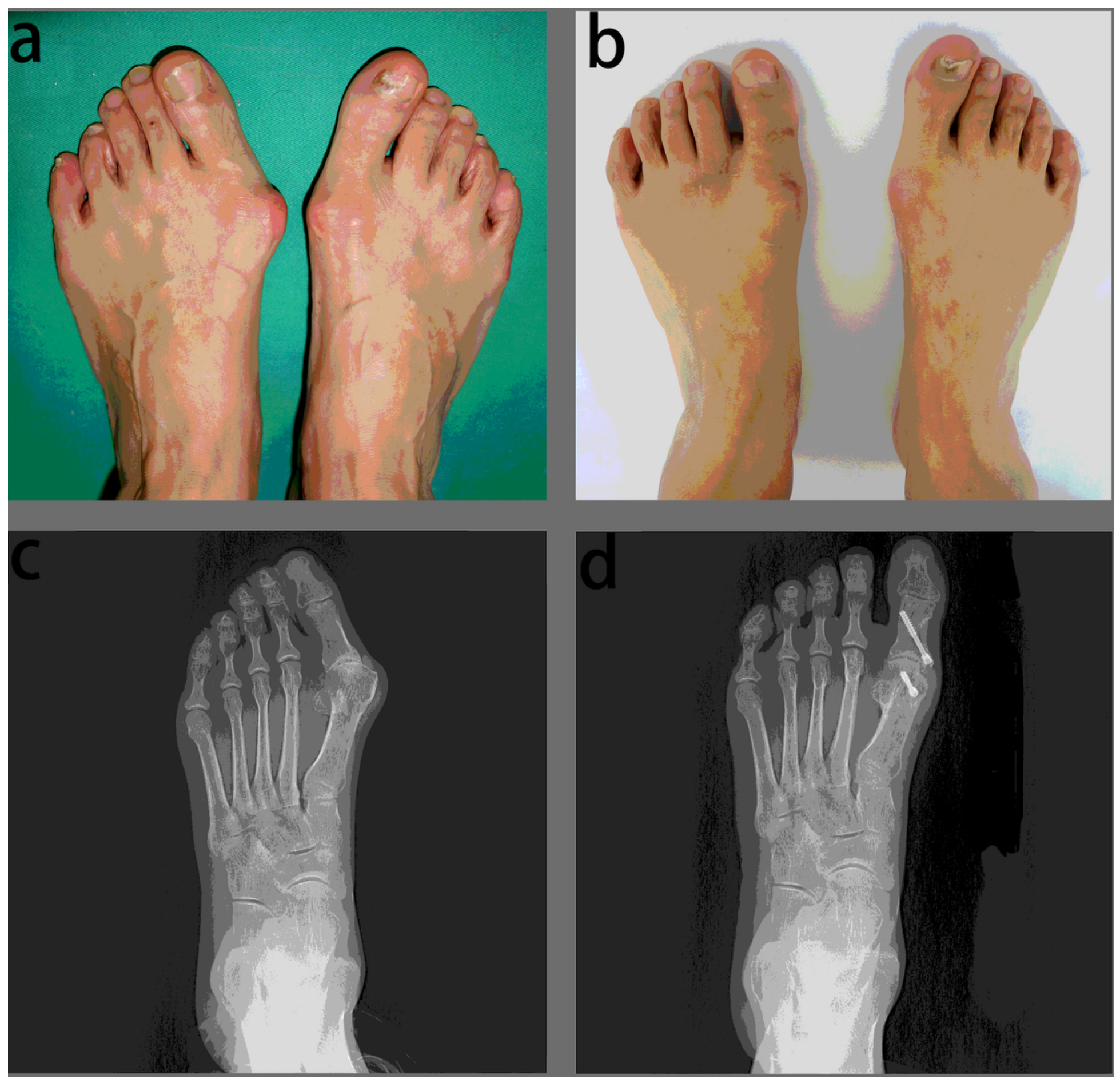

Figure 4

Clinical and radiographic view of a 60-year-old female with a left hallux valgus. Preoperative clinical photo: preoperative(a) and postoperative 24 months follow-up (b); radiographic X-ray: preoperative (c) and postoperative 24 months follow-up (d). 

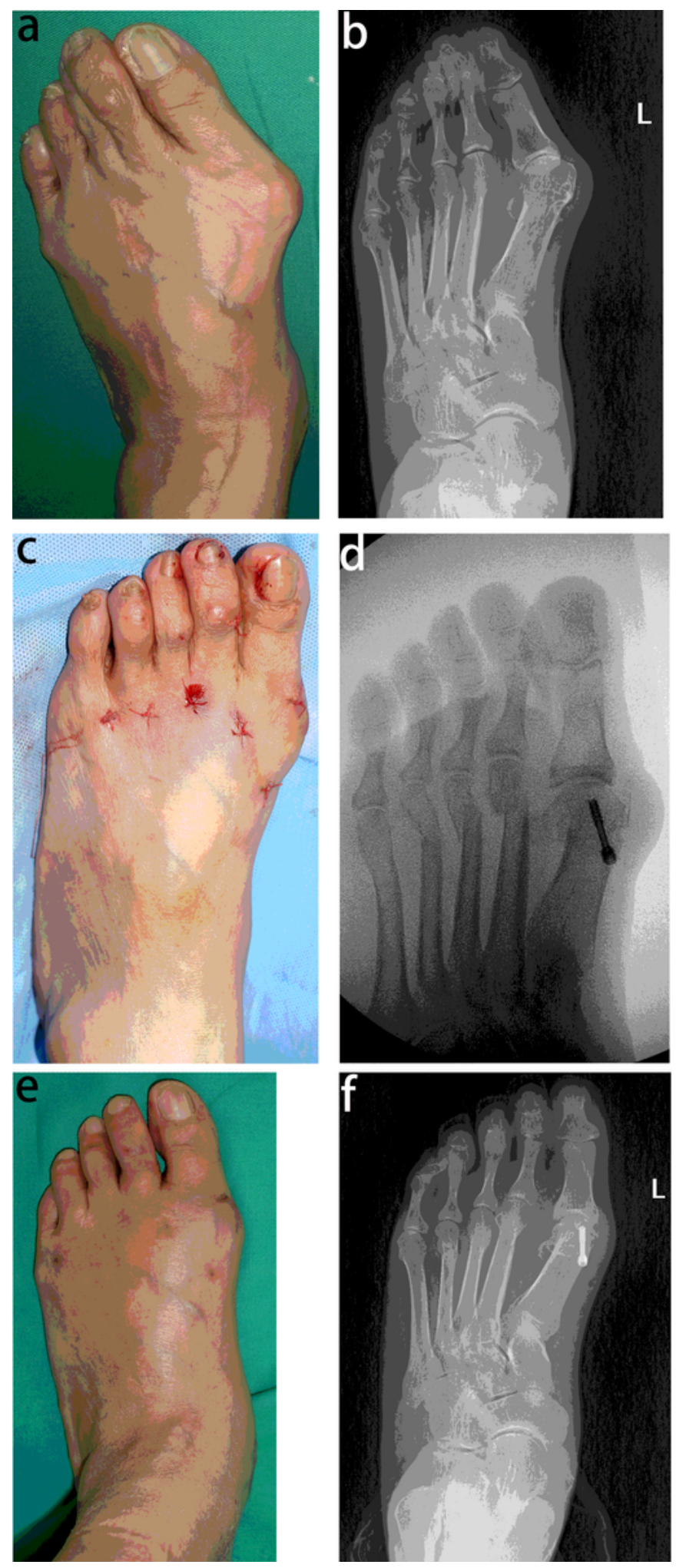

\section{Figure 5}

Clinical and radiographic view of a 67-year-old female with a left hallux valgus. Preoperative clinical photo(a) and radiographic X-ray(b). The minimal clinical incision (c) and radiographic X-ray showing the osteotomy of 2-4 metatarsals percutaneously without fixation (d) during the operation. Postoperative clinical photo(e) and radiographic X-ray (f) at 16 months follow-up. 


\section{Supplementary Files}

This is a list of supplementary files associated with this preprint. Click to download.

- firstmetatarsalosteotomy.MP4 